\title{
Imaging in Lyme neuroborreliosis
}

\author{
Elisabeth S. Lindland ${ }^{1,2,3}$ - Anne Marit Solheim ${ }^{4,5} \cdot$ Silje Andreassen $^{3,6}$ - Else Quist-Paulsen ${ }^{3,7}$ - Randi Eikeland ${ }^{4,8}$. \\ Unn Ljøstad ${ }^{4,5}$ • Åse Mygland ${ }^{4,5} \cdot$ Ahmed Elsais $^{9}$ • Gro O. Nygaard ${ }^{9}$ • Åslaug R. Lorentzen ${ }^{4,8}$ • Hanne F. Harbo ${ }^{3,9}$. \\ Mona K. Beyer ${ }^{1,10}$
}

Received: 2 May 2018 / Revised: 25 June 2018 / Accepted: 4 July 2018 / Published online: 4 September 2018

(C) The Author(s) 2018

\begin{abstract} article can educate those engaged in imaging of the nervous system and serve as a comprehensive tool in clinical cases.

\section{Key Points}

- Diagnostic criteria for LNB emphasise exclusion of an alternative cause to the clinical symptoms.

- MRI makes a crucial contribution in the diagnosis and follow-up of LNB.

- MRI may have normal findings, or show neuritis, meningitis, myelitis, encephalitis or vasculitis.

- White matter lesions are not a prominent feature of LNB.
\end{abstract}

Lyme neuroborreliosis (LNB) is a tick-borne spirochetal infection with a broad spectrum of imaging pathology. For individuals who live in or have travelled to areas where ticks reside, LNB should be considered among differential diagnoses when clinical manifestations from the nervous system occur. Radiculitis, meningitis and facial palsy are commonly encountered, while peripheral neuropathy, myelitis, meningoencephalitis and cerebral vasculitis are rarer manifestations of LNB. Cerebrospinal fluid (CSF) analysis and serology are key investigations in patient workup. The primary role of imaging is to rule out other reasons for the neurological symptoms. It is therefore important to know the diversity of possible imaging findings from the infection itself. There may be no imaging abnormality, or findings suggestive of neuritis, meningitis, myelitis, encephalitis or vasculitis. White matter lesions are not a prominent feature of LNB. Insight into LNB clinical presentation, laboratory test methods and spectrum of imaging pathology will aid in the multidisciplinary interaction that often is imperative to achieve an efficient patient workup and arrive at a correct diagnosis. This

Keywords Lyme neuroborreliosis $\cdot$ MRI $\cdot$ Neuritis $\cdot$ Myelitis $\cdot$ Encephalitis

$\begin{array}{ll}\text { Abbreviations } \\ \text { AAN } & \text { American Academy of Neurology } \\ \text { ADC } & \text { Apparent diffusion coefficient } \\ \text { AI } & \text { Antibody index } \\ \text { Bb } & \text { Borrelia burgdorferi }\end{array}$

Abbreviations

AAN

AI

$\mathrm{Bb}$

Elisabeth S. Lindland

elisalindland@gmail.com

1 Department of Radiology and Nuclear Medicine, Oslo University Hospital, Sognsvannsveien 20, 0372 Oslo, Norway

2 Department of Radiology, Sorlandet Hospital, Sykehusveien 1, N-4809 Arendal, Norway

3 Institute of Clinical Medicine, University of Oslo, Oslo, Norway

4 Department of Neurology, Sorlandet Hospital, Kristiansand, Norway

5 Institute of Clinical Medicine, University of Bergen, Bergen, Norway
CLIPPERS Cenhancement responsive to steroids

CNS Central nervous system

CSF Cerebrospinal fluid

EFNS European Federation of Neurological Societies

FLAIR Fluid attenuated inversion recovery 


$\begin{array}{ll}\text { LNB } & \text { Lyme neuroborreliosis } \\ \text { MRI } & \text { Magnetic resonance imaging } \\ \text { MS } & \text { Multiple sclerosis } \\ \text { PET } & \text { Positron emission tomography } \\ \text { SPECT } & \text { Single photon emission computed tomography } \\ \text { TBE } & \text { Tick-borne encephalitis }\end{array}$

\section{Introduction}

Lyme borreliosis is an infection with the spirochete Borrelia burgdorferi ( $\mathrm{Bb}$ ). It is transmitted through tick bites, and is the most common vector-borne disease in Europe and North America [1, 2]. Estimates of prevalence are 100-130 per 100,000 in Europe, and 20-100 cases per 100,000 people in the United States (US) [3]. Involvement of the nervous system is reported to occur in 10-15\% of patients with borreliosis [4].

In Europe, the dominant strains are Borrelia garinii and Borrelia afzelii, whereas Borrelia burgdorferi sensu stricto is the only pathogen in American Lyme borreliosis [4-6].

Lyme neuroborreliosis (LNB) is an important differential diagnosis in patients with nonspecific nervous system MRI findings and possibility of previous exposure to tick bite. In this article, we explain the diagnostic criteria, summarise and discuss current knowledge of imaging and pathology in LNB and describe the imaging findings in a spectrum from the typical presentations (meningoradiculitis, cranial nerve involvement), to less common (myelitis, peripheral neuropathy) and rare disease courses (meningoencephalitis, vasculitis).

\section{Disease manifestations}

The clinical presentation of LNB can vary widely, partly due to the genetic differences in spirochetal strains. Individual variations in immunological response and the possibility of co-infections may also explain the wide spectrum of symptoms that can be encountered. The skin is primarily affected, and erythema migrans can be observed. About one third to half of patients with LNB recall a tick bite or a rash $[7,8]$. The most common neurological manifestation of LNB in adults is the Bannwarth syndrome with painful radiculoneuritis and aseptic meningitis, the former is most prominent in Europe and the latter in North America [1]. LNB with facial nerve palsy is commonly encountered in both continents. Rarer manifestations from the nervous system are meningoencephalitis, encephalomyelitis, cerebral vasculitis and peripheral neuropathy [6]. LNB may rarely present to the ophthalmologist with optic neuropathies and papilledema due to raised intracranial pressure [9]. The distinction between early and late LNB is made from duration of neurological symptoms of less or more than 6 months, and more than $95 \%$ of cases are classified as early disease [10]. Most LNB patients
Table 1 EFNS diagnostic criteria for $\operatorname{LNB}^{\mathrm{a}}[10]$

\begin{tabular}{lcl}
\hline Criteria & $\begin{array}{l}\text { Definite } \\
\text { LNB }\end{array}$ & Possible LNB \\
\hline $\begin{array}{l}\text { 1) Neurological symptoms } \\
\text { suggestive of LNB without } \\
\text { other obvious reasons }\end{array}$ & $\begin{array}{c}\text { All three } \\
\text { criteria } \\
\text { fulfilled }\end{array}$ & $\begin{array}{c}\text { Clinical criteria and one of } \\
\text { two laboratory criteria } \\
\text { fulfilled }\end{array}$ \\
$\begin{array}{l}\text { 2) Cerebrospinal fluid } \\
\text { pleocytosis }\end{array}$ & & \\
$\begin{array}{l}\text { 3) Intrathecal } B b \text { antibody } \\
\text { production }\end{array}$ & & \\
\hline
\end{tabular}

EFNS European Federation of Neurological Societies, LNB Lyme neuroborreliosis

${ }^{\text {a }}$ Special criteria apply for late LNB with polyneuropathy [10]

${ }^{\mathrm{b}}>5$ white blood cells per $\mathrm{mm}^{3}$

respond well to antibiotic treatment, but some experience persistent complaints [11, 12]. A definition for post-Lyme disease syndrome has been suggested to include subjective symptoms of fatigue, cognitive symptoms and/or widespread musculoskeletal pain starting within 6 months after completed treatment [13]. Post-Lyme disease syndrome is not discussed in this article.

\section{Diagnostic criteria}

The European Federation of Neurological Societies (EFNS) established guidelines for LNB diagnosis in 2010 [10]. The criteria for LNB diagnosis are: LNB compatible neurological symptoms and signs with no other explanation, pleocytosis and production of $B b$ specific antibodies in the cerebrospinal fluid (CSF). Intrathecal antibody production is often expressed as a positive $B b$ antibody index (AI), where measurements of antibody show a higher level in CSF than in serum [14]. The European guidelines state that the condition is possible when two out of three criteria are present, and definite when all three criteria are met (Table 1). Direct test methods for detection of $B b$, mainly culture and polymerase chain reaction, have low sensitivity and are seldom helpful in the diagnostic work-up [14]. Interpretation of serology can also be a challenge in the diagnostic process, since the sensitivity is low in the early stage of infection. Further, both IgM and IgG antibodies can remain positive several years after infection, so results may not differentiate previous exposure, reinfection or acute infection $[14,15]$. American Academy of Neurology (AAN) diagnostic criteria do not include analysis of cerebrospinal fluid, but presence of one or more of erythema migrans, direct proof through histopathology, microbiology or polymerase chain reaction or immunologic evidence of infection [4]. To increase specificity of serology, a two-tier approach is recommended with an initial enzyme immunoassay followed by separate immunoglobulin Western blots if the first step is positive [14]. Both American and European diagnostic 
criteria for LNB emphasise the exclusion of other apparent cause to the clinical disorder [4]. Magnetic resonance imaging (MRI) therefore makes a crucial contribution in the diagnosis and follow-up of LNB.

\section{Pathology}

A recent study with inoculation of $B b$ infection in the central nervous system (CNS) of rhesus macaques resulted in
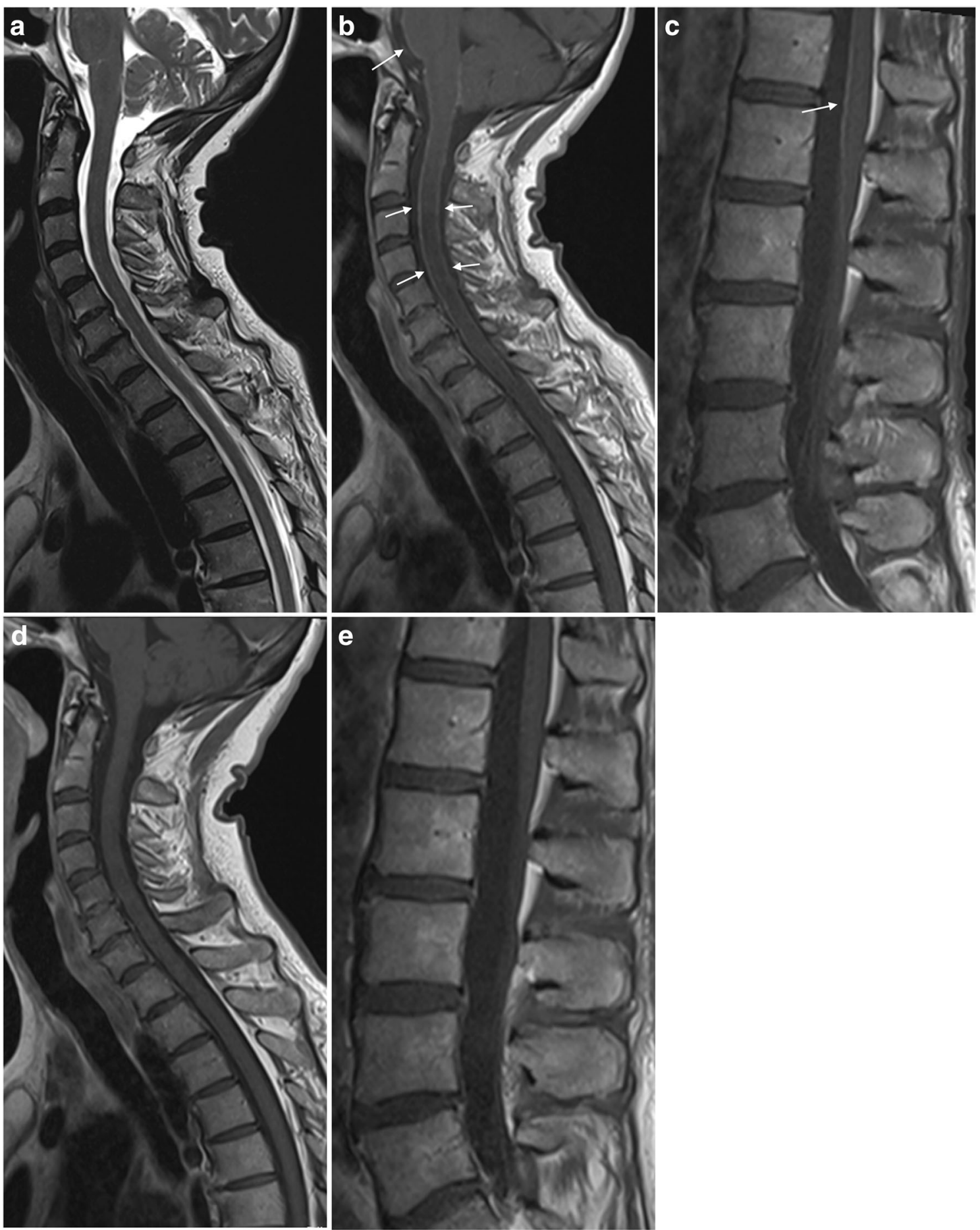

Fig. 1 Meningoradiculitis due to Lyme neuroborreliosis (LNB) in a 73year-old female. She experienced periods of strong superficial abdominal pain, reduced appetite and headache. Sagittal T2 (a) and T1 after gadolinium-based contrast agent injection $(\mathbf{b}, \mathbf{c})$ demonstrated normal cord parenchyma and diffuse leptomeningeal enhancement (arrows) of spinal cord and brain stem. Cerebrospinal fluid (CSF) analysis showed pleocytosis and intrathecal production of Borrelia burgdorferi $(B b)$ specific IgM and $\operatorname{IgG}$ antibodies. No leptomeningeal enhancement was found 4 weeks after the treatment on post-contrast sagittal T1 weighted images $(\mathbf{d}, \mathbf{e})$ 
lymphocytic neuritis and ganglionitis, and neuronal degeneration and demyelination in the peripheral nervous system. In brainstem and medulla there were lesions with focal malacia and nerve fibre degeneration, and some subjects had myelitis with necrosis and degeneration [16]. The experiment gave evidence that the nerve injuries are mainly due to immune mediated mechanisms. Human studies are limited, but have pointed mainly to axonal damage in LNB mononeuropathy and plexopathy $[4,17]$. The myelitis seen in most LNB cases could be the inflammation of radiculoneuritis extending to the spinal cord, CNS parenchymal involvement is otherwise considered rare in LNB [4]. $B b$ invasion into the human brain has been reported in four cases with biopsy from MRI enhancing lesions [18-20].

\section{Imaging in Lyme neuroborreliosis}

Early imaging reports, starting with Halperin et al. in 1988 and 1989, focused on non-specific white matter changes in Lyme encephalopathy, considered a late phase of LNB with mild cognitive deficits $[21,22]$. These and six other studies published between 1990 and 2007 included 10-27 subjects, and white matter lesions were found in $15-63 \%$ of the patients [23-28]. The diagnostic criteria in these reports varied, and for many cases would not fulfil the EFNS or AAN criteria of today. Sequence development and higher resolution of MRI have constantly evolved with increased sensitivity of this imaging method, and knowledge of age-related white matter changes has also changed throughout this time. Therefore, the claim that LNB causes non-specific white matter changes should be considered based on weak scientific evidence. In 2009 Agarwal and Sze reported imaging data in 66 patients with LNB. Seven patients, but also six out of the 50 healthy control subjects, had white matter lesions, and the result suggests that white matter lesions are not a feature of LNB. One patient had an enhancing parenchymal lesion and three had meningeal or cranial nerve enhancement [18]. Numerous case reports demonstrate that LNB has a wide spectrum of imaging entities, including diffuse or tumour-like affection in brain or spinal cord [19, 20, 29-34], meningeal and/or nerve enhancement [33-43], as well as vascular affection with stroke-like presentation [44-54] or haemorrhage [35, 55, 56]. Overlap of imaging
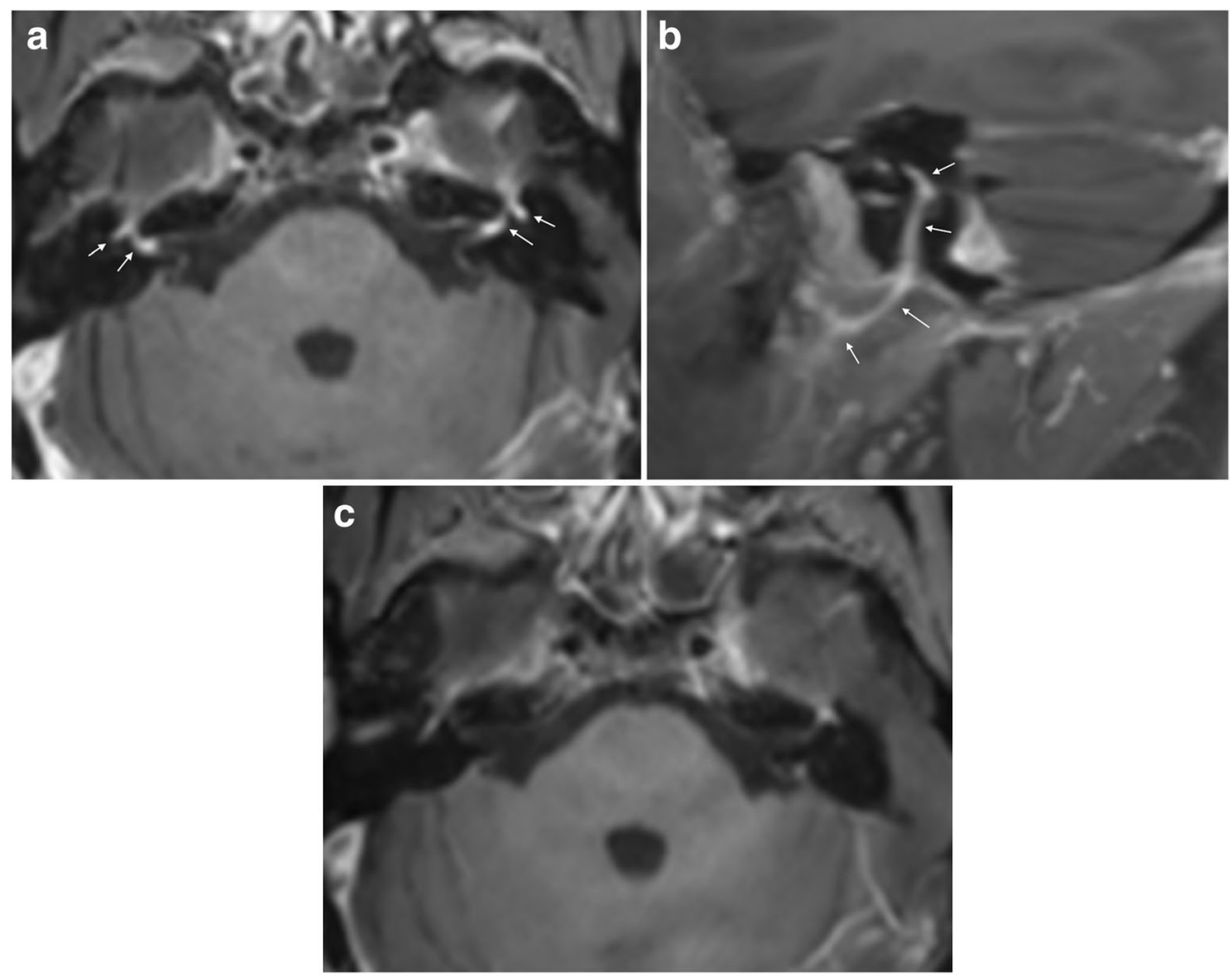

Fig. 2 Facial neuritis in LNB with typical disease course in a 55-year-old female. She suffered from back pain radiating to chest, arms, head and neck, especially intense at night time, and after 2 weeks she also developed bilateral facial palsy. CSF analysis showed 594 cells $/ \mathrm{mm}^{3}$ (normal range $0-5 / \mathrm{mm}^{3}$ ) and production of $B b$ specific $\mathrm{IgG}$ and $\mathrm{IgM}$ antibodies. Axial post-contrast fat suppressed T1-weighted image (a) showed intense enhancement in the distal intracanalicular, labyrinthine and geniculate ganglion segments of both facial nerves (arrows point to these structures which are mentioned from medial to lateral). $3 \mathrm{~mm}$ sagittal reformat (b) on the right side demonstrated the enhancement involving the tympanic and mastoid segment, as well as the parotid segment with the temporofacial and cervicofacial branches at the pes anserinus included (arrows point to these structures which are mentioned from cranial to caudal). Axial post-contrast fat suppressed T1-weighted image showed no abnormal enhancement of facial nerves 6 months after the treatment $(\mathbf{c})$ 
Fig. 3 LNB myelitis in a 35-year old female who presented with pain radiating in the right arm, neck stiffness and general symptoms of headache, fatigue, nausea and vomiting. T2 weighted images showed subtle spinal cord hyperintensity, on sagittal image (a) from C2-C5 centrally located (arrows), and on axial $\mathrm{T} 2$ gradient echo image at the $\mathrm{C} 2 / \mathrm{C} 3$ level (b), dominant affection adjacent to the right anterior horn was seen (arrow). CSF analysis showed increased cell count as well as intrathecal production of $B b$ specific IgM and $\mathrm{IgG}$ antibodies. Two months after treatment she had fully recovered. Seven years has passed since her symptom debut, and there has been no further incidence of neurological affection

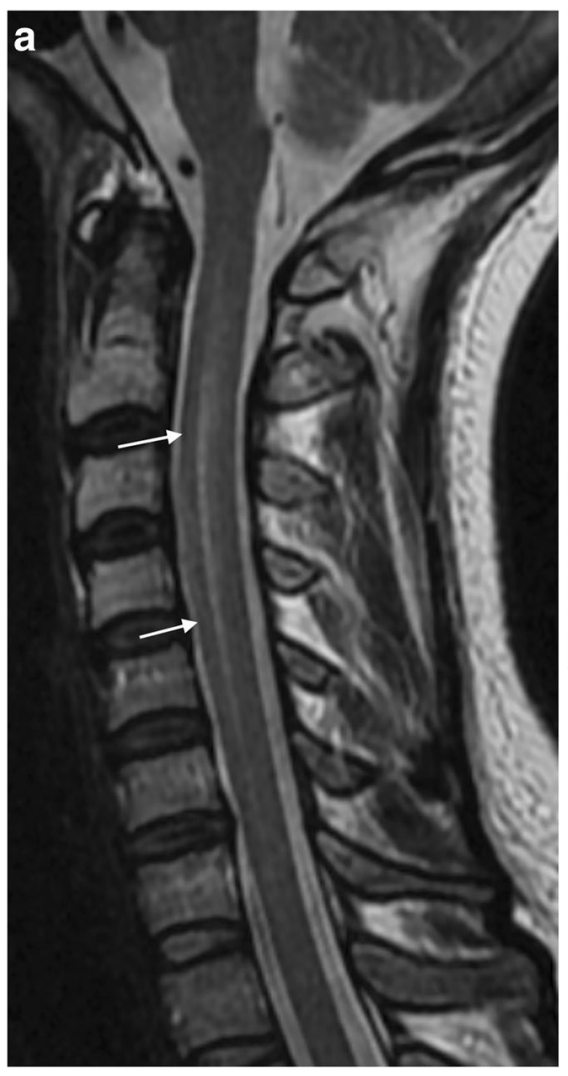

findings between LNB and multiple sclerosis (MS) has been discussed [1, 57], but is understudied.

There is limited knowledge of structural and functional changes in LNB with quantitative MRI techniques. Three studies have reported structural changes, and no difference between the patients and controls was found [23, 27, 28]. However, these studies included a limited number of subjects and controls $(n=6-20)$, so they probably lacked power to show subtle changes. Functional studies have been conducted with PET $[58,59]$ and with SPECT $[26,60-62]$. These studies reported regional hypoactivity and hypoperfusion, but were hampered by heterogeneous study populations with non-specific symptoms and included individuals with uncertain diagnoses. Larger, prospectively conducted case-control studies are needed to learn more about imaging abnormalities in LNB.

\section{Meningoradiculitis}

The most typical manifestation of nervous system involvement in European Lyme borreliosis is the Bannwarth syndrome with radiculoneuritis, causing radicular pain and sometimes paresis of
Fig. 4 Brachial plexus neuritis in a 74-year-old woman with acute distal right arm paresis. In the preceding weeks she had nausea, vomiting and weight loss, and felt increasingly forgetful and unsteady. A fluid-sensitive coronal STIR sequence showed diffusely increased signal of the nerve bundle on the right side (arrows) compared to the left side. Lumbar puncture followed, and showed cell count of $101 / \mathrm{mm}^{3}$ as well as a positive $B b \mathrm{IgG}$ antibody index (AI)

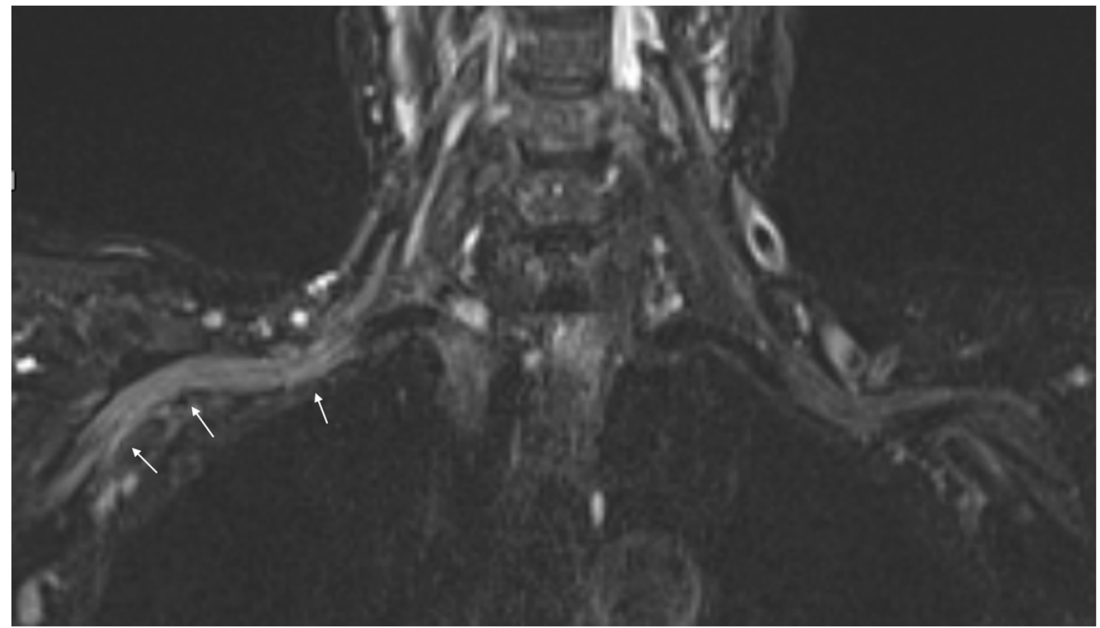



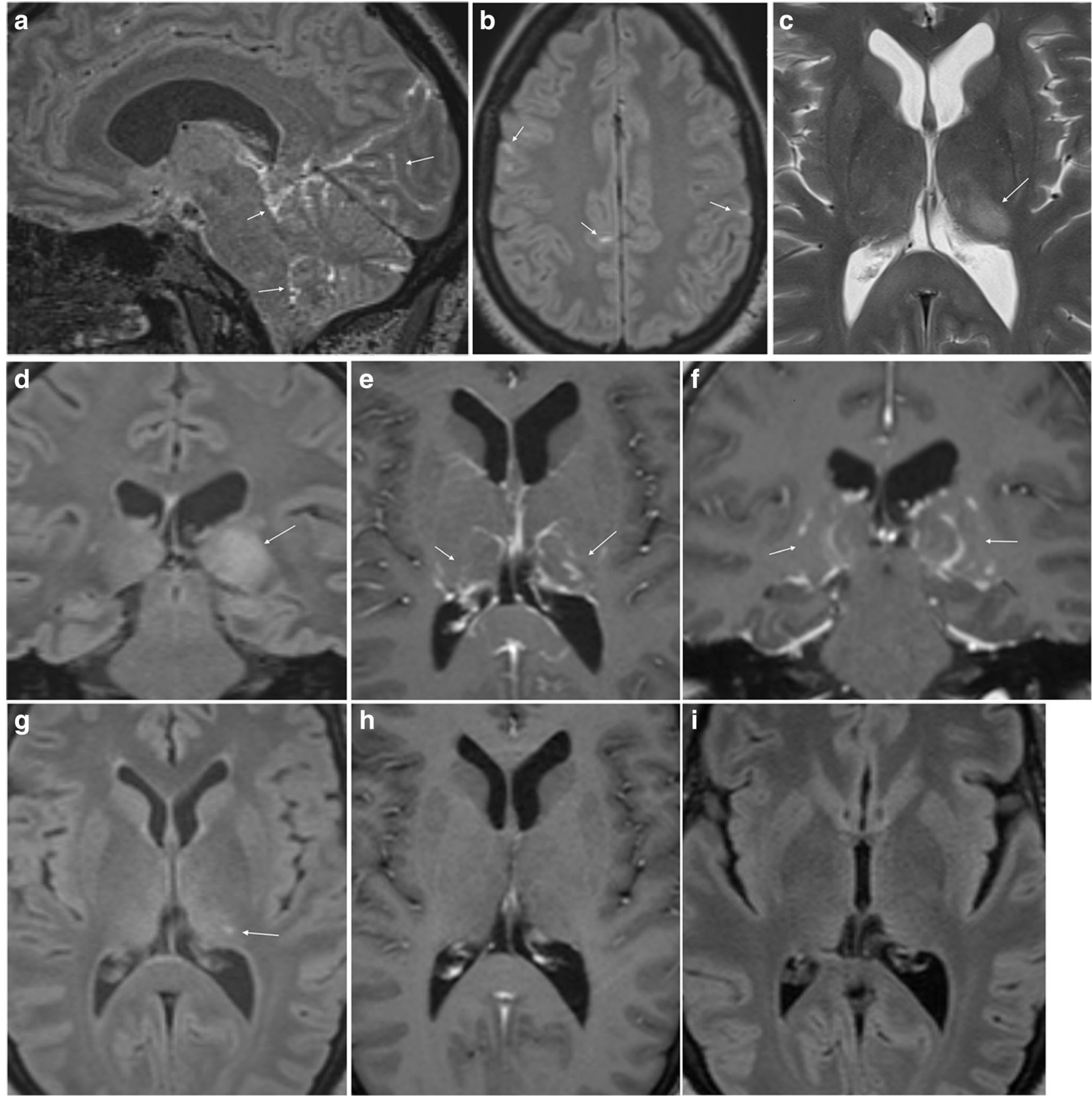

Fig. 5 LNB meningoencephalitis in a 45-year-old female who presented with fatigue, headache, dizziness and vomiting. Neurological findings were tremor and unsteady gait. The CSF cell count was 270 cells $/ \mathrm{mm}^{3}$ and there was a positive $B b$ IgG AI. Post-contrast sagittal (a) and axial (b) FLAIR images demonstrated patchy leptomeningeal enhancement over the cerebral convexities and in the posterior fossa (arrows). There was a hyperintense lesion in the left thalamus (arrow) on axial T2-weighted image (c) and coronal FLAIR image (d). There was no abnormality in

extremities or the abdominal wall $[1,7]$. Patients often describe nocturnal exacerbation of the pain, and location and intensity may vary [10]. Studies report $19.5-29 \%$ of LNB cases to have clinical signs of meningitis $[7,8]$. Enhancement of meninges, cranial and spinal nerves can be encountered. Images in Fig. 1 the lesion on diffusion weighted images (not shown). Post-contrast axial (e) and coronal (f) T1-weighted images showed punctate and curvilinear uptake (arrows) in the thalamic lesion. Similar, subtle changes were present in the right thalamus. After 2 months there was only slight residual hyperintensity in the left thalamus (arrow) on axial FLAIR image (g) and no sign of enhancement on post-contrast axial T1 weighted image (h). One year after treatment there were no imaging abnormalities (i)

show a case of LNB with meningoradiculitis where leptomeningeal enhancement was diffuse and widespread, and disappeared promptly with treatment. The frequency of leptomeningeal enhancement on MRI examination in LNB has only been reported in the above mentioned retrospective study by 

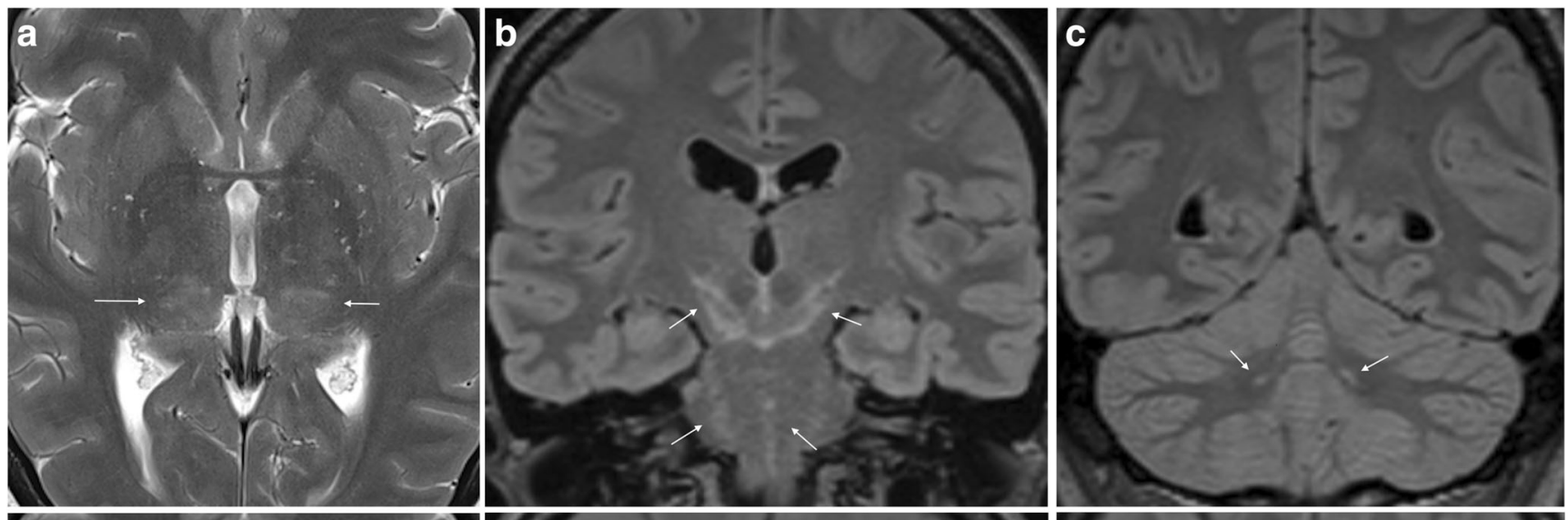
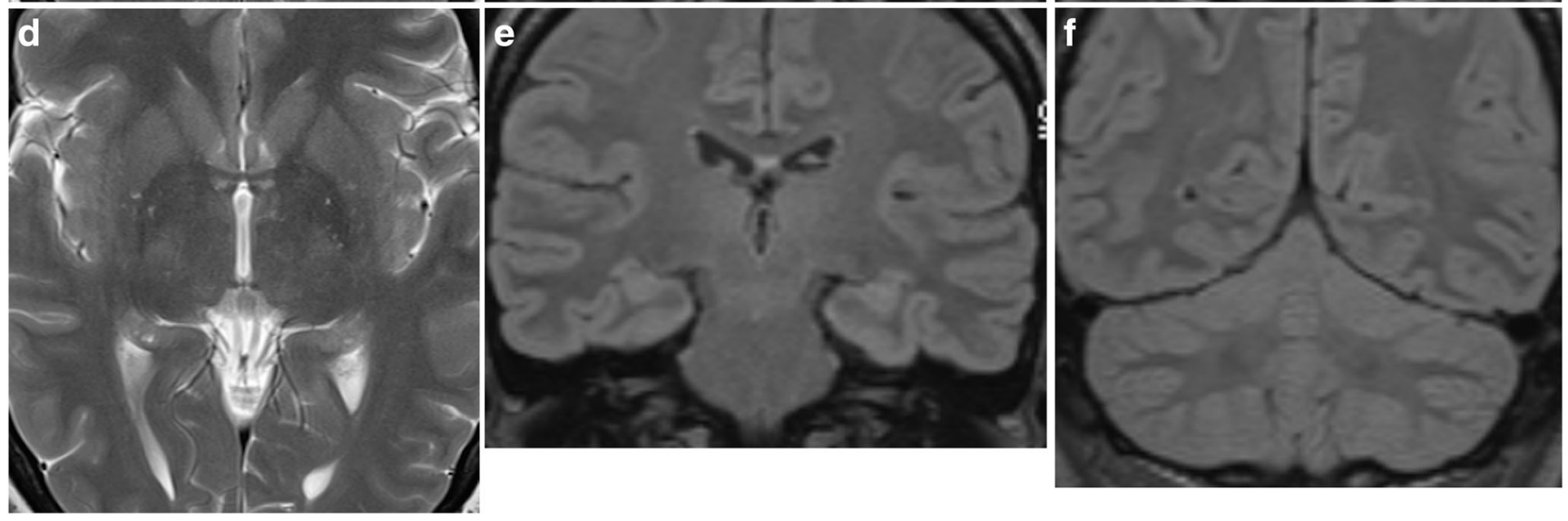

Fig. 6 LNB encephalitis in a 40-year-old woman. She presented with 1 day history of headache, vomiting, dizziness and personality change. The CSF cell count was 182 cells $/ \mathrm{mm}^{3}$. MRI showed symmetric diffuse and patchy T2 (a) and FLAIR (b, c) hyperintensity of inferior thalami (arrows in a), cerebral peduncles, mesencephalon, pons (arrows in b) and cerebellar dentate nuclei (arrows in $\mathbf{c}$ ). There were no abnormalities on

Agarwal and Sze. One of 63 LNB cases with contrast enhanced MRI examination in that study showed mild leptomeningeal enhancement [18]. For bacterial meningitis in general, postcontrast T1-weighted images yield a low rate of leptomeningeal enhancement, while the use of post-contrast FLAIR sequence increases sensitivity of MRI [63, 64]. It is also important to be aware of the rather low specificity of meningeal enhancement with T1-weighted images, mainly due to interpretation disturbed by the normal enhancement in vascular structures [65].

\section{Cranial neuritis}

About $80 \%$ of cranial nerve involvement in LNB affect facial nerves, bilaterally in $25 \%$ of the cases $[4,66]$. In a recently published retrospective evaluation of $68 \mathrm{LNB}$ patients, facial palsy was the cause for hospitalisation in $50 \%$ of the patients [8]. A study by Ogrinc et al. of 77 patients with early LNB reported facial palsy in 36\% [7]. MRI can demonstrate diffuse enhancement of cranial nerves, as shown in Fig. 2. There are no reports of diffusion-weighted or post-contrast images (not shown). Further clinical and laboratory investigations revealed erythema migrans on the right thigh and a positive CSF $B b$ IgG AI. Tests for TBE antibodies were negative. She received antimicrobial treatment, and repeated MRI 8 weeks after the initial scan was normal $(\mathbf{d}-\mathbf{f})$

nodular enhancement. Imaging evaluation of inflammation in the facial nerve can be challenging due to the lack of specificity concerning aetiology, in addition to normal enhancement seen in long segments of this nerve [67]. In the clinical setting, attention should be paid to asymmetry or marked intensity of enhancement in the geniculate ganglion, tympanic or mastoid segments, while there is normally no enhancement in the cisternal, intracanalicular, labyrinthine or parotid segments. To our knowledge, there are no data of the diagnostic accuracy of MRI in LNB facial neuritis. In 36 patients with idiopathic facial palsy a high sensitivity (100\%) was found in contrast enhanced 3D T1 gradient echo sequence with fat suppression, and specificity was improved from 57 to $86 \%$ with the use of a contrast enhanced 3D FLAIR sequence [68]. In the workup of facial palsy, MRI can exclude a compressing lesion, brainstem infarct, perineural tumour spread or focal lesion such as schwannoma [67]. Certain concomitant MRI findings can be suggestive of some inflammatory etiologies such as Guillain-Barré syndrome, multiple sclerosis and sarcoidosis. In most cases of facial palsy, CSF 

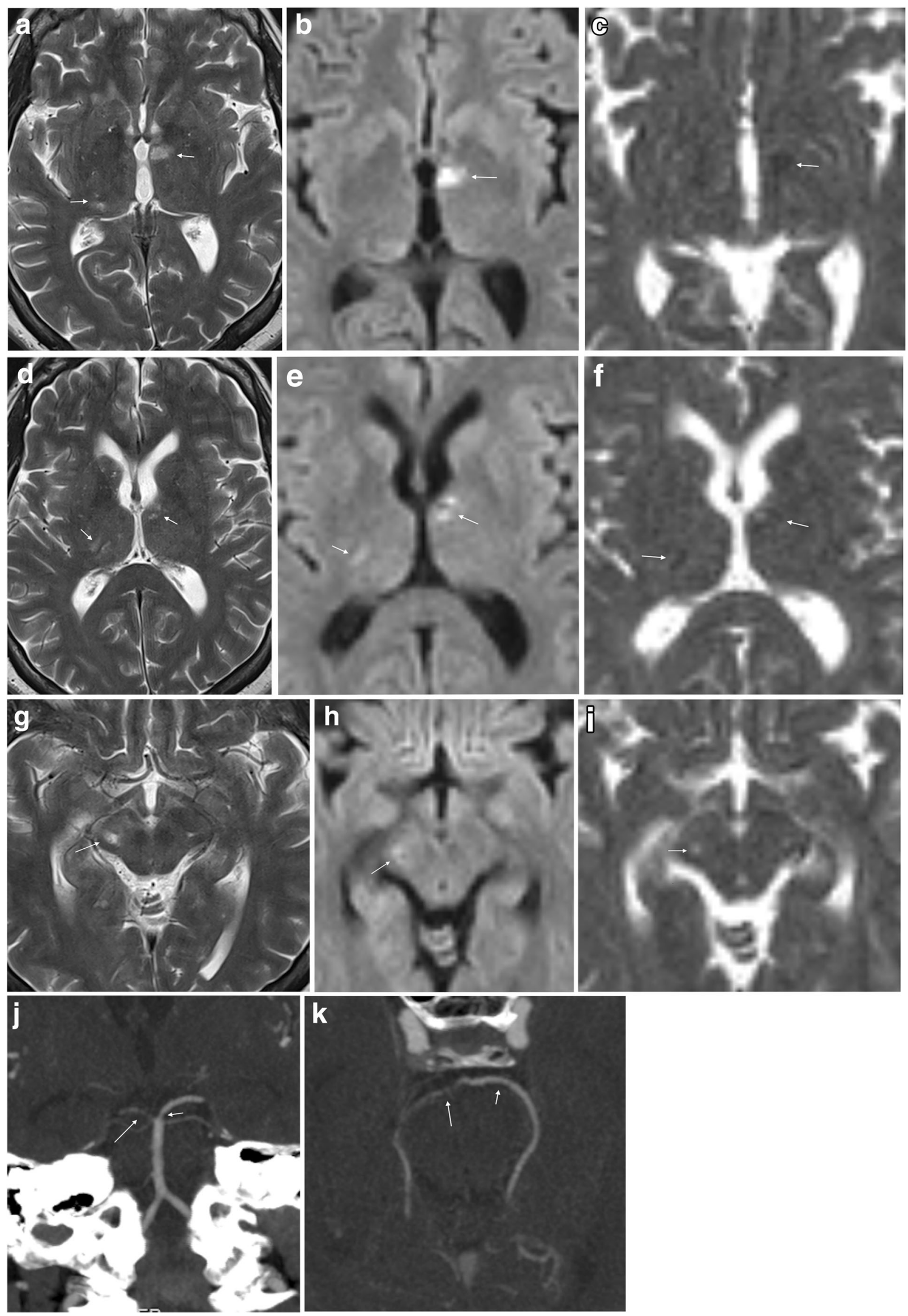
4 Fig. 7 LNB vasculitis in a 55-year-old woman with strange behaviour for 3 days preceding hospital admission. She had incoherent speech and was disorientated. CSF cell count was 213 cells $/ \mathrm{mm}^{3}$ and $B b \mathrm{IgG}$ AI was positive. MRI revealed ischemic lesions of different ages in thalamus bilaterally (a-f), right cerebral peduncle $(\mathbf{g}-\mathbf{i})$ and right occipital cortex (not shown). Axial T2-weighted images (a, d, g), diffusion weighted B1000 images $(\mathbf{b}, \mathbf{e}, \mathbf{h})$ and $\operatorname{ADC}$ maps $(\mathbf{c}, \mathbf{f}, \mathbf{i})$ demonstrated the lesions. In contrast to the hypointense lesions on ADC maps in the thalami (arrows in $\mathrm{c}$ and $\mathrm{f}$ ) consistent with cytotoxic edema in recent infarcts, the lesion in the right cerebral peduncle was hyperintense on both B1000 image (arrow in h) and ADC map (arrow in i), consistent with vasogenic edema and a more advanced stage of ischemic lesion. $5 \mathrm{~mm}$ MIP coronal (j) and axial (k) reformats of CT angiography examination demonstrated several short stenotic segments of both posterior cereberal arteries (arrows), most pronounced on the right side

analysis is an important supplemental test that will help to differentiate, and possibly identify, inflammatory and infectious etiologies.

\section{Myelitis}

Myelitis confirmed by MRI constituted 7\% of the LNB manifestations leading to hospitalisation in the aforementioned study by Schwenkenbecher et al. [8]. In addition to the five cases from that study, we have found 11 adult case reports of LNB myelitis with abnormal MRI findings of the spinal cord substance [29, 34, 42, 69-76]. In all of them the cervical spinal cord was affected. The majority were, like the case presented in Fig. 3, longitudinally extensive and centrally or slightly anteriorly located. One case had a focal lesion at the C5 level [69], and another multifocal cervical lesions [75]. Enhancement patterns ranged from no enhancement, to nodular or diffusely extensive contrast enhancement. Also, one case report of a cervical spinal cord syrinx exists [73]. Lumbosacral myelitis and normal MRI findings have also been reported [39, 77]. In the setting of an acute transverse myelitis, additional findings of nerve root or leptomeningeal enhancement can be helpful imaging features in differentiating LNB from MS and other causes of centrally located myelitis such as acute disseminated encephalomyelitis and neuromyelitis optica spectrum disorders. Many infectious myelopathies can have combined manifestations with meningitis, radiculitis and encephalitis, among these are varicella-zoster virus, Epstein-Barr virus, tick-borne encephalitis (TBE) virus and cytomegalovirus [78]. History of pain can also be an important clue to the LNB diagnosis, but again CSF analysis is the key supplemental test.

\section{Peripheral neuropathy}

Bannwarth syndrome with radicular pain and/or facial palsy are typical LNB manifestations. Plexus neuritis or mononeuritis multiplex are other peripheral manifestations, seen in 5-10\% of LNB cases [10]. This can present as a chronic asymmetric neuropathy, and will in such cases usually not have meningitis or intrathecal antibody production [79]. In neuritis due to LNB, nonspecific findings such as increased signal of nerve structures on fluid-sensitive sequence can be encountered. Figure 4 shows a case of acute peripheral motor nerve affection due to LNB brachial plexus neuritis. No symptoms of radiculitis preceded the motor affection in our patient, which is untypical $[29,80]$. Due to the focal or regional distribution of symptoms in such patients, MRI will commonly supplement clinical, blood and electrodiagnostic tests, in case nerve entrapment or a mass lesion can be revealed [81].

\section{Encephalitis}

Among the 68 patients studied by Schwenkenbecher et al., one adult and two children were clinically classified as acute encephalitis, and one of them had abnormal MRI findings [8]. Non-specific MRI findings of diffuse involvement of brain parenchyma are among the few case reports mentioned earlier, and has also occasionally been encountered in our institutions (Figs. 5 and 6). The case in Fig. 5 has a resemblance to CLIPPERS (chronic lymphocytic inflammation with pontine perivascular enhancement responsive to steroids), an entity thought to represent immune-mediated inflammation [82]. Interestingly, in our patient the abnormality resolved with antibiotic treatment only. Massengo et al. reported a case with similar type of punctate and linear enhancing lesions in cerebral and cerebellar parenchyma which responded to steroid treatment [32]. No specific pattern of involvement for LNB encephalitis can be identified from the few cases that exist. Still, of note is that it seems closer to the involvement pattern seen in rhombencephalitis, which also can present in combination with leptomeningeal enhancement in infections such as Listeria monocytogenes and tuberculosis. Involvement of insula, temporal lobe and limbic system, which is typical for herpes simplex virus infection, has not been reported in LNB [83]. We have seen cases with involvement of thalami, and this was also reported in a case of LNB encephalitis by Haene et al. [30]. Among other conditions with acute and subacute thalamic affection are Japanese encephalitis, acute disseminated encephalomyelitis, neuro-Bechet, venous and arterial infarctions. Involvement of thalami, basal ganglia and cerebellum have also been proposed to be suggestive of viral tickborne encephalitis [84, 85]. Tests for specific antibodies to both $B b$ and TBE virus should be included in the workup of patients with suspected encephalitis who live in or have travelled to areas where these tick-borne infections are endemic. 


\section{Cerebral vasculitis}

LNB vasculitis is presumed to be caused by the inflammatory response to the spirochetal infection [16]. A German study calculated a $0.3 \%$ chance of cerebral vasculitis in Lyme borreliosis, and there may be an affinity for involvement of the posterior cerebral circulation in LNB vasculitis $[44,86]$. Imaging is necessary to recognise this type of complication (Fig. 7). Diffusion weighted imaging can identify the restricted diffusion typical for acute and subacute ischemic lesions, a finding which has not been reported or seen in our experience in LNB encephalitic lesions. Angiographic examinations can demonstrate irregularities of the vessel lumen with occlusion or segmental narrowing or dilatation. Such findings support a diagnosis of vasculitis, and can be attributed to LNB in case of fulfilled diagnostic criteria as described previously. It is important for radiologists and clinicians to be aware of LNB as a possible cause for stroke and vasculitis in patients exposed to ticks.

\section{Conclusion}

Non-specific imaging findings are a constant challenge to radiologists. Insight into clinical disease courses and laboratory test methods will aid in the multidisciplinary interaction that often is imperative to achieve an efficient patient workup and arrive at a correct diagnosis. This is especially true in LNB, where a wide spectrum of neurological, and sometimes general and cognitive symptoms can be encountered, as well as diverse imaging pathology from neuritis to meningitis, encephalitis and vasculitis. The primary role of imaging is to look for other causes to explain the clinical symptoms. This article can educate those engaged in imaging of the nervous system and serve as a comprehensive tool when LNB is in question.

Acknowledgements Thanks to Christian G. Lund, Anne-Helene Moksness and Karen Herlofsson for conveying and discussing cases, Harald Reiso for management of the BorrSci project, and to the patients for permission to publish.

Funding Research fellowship for Elisabeth S. Lindland, Anne Marit Solheim and Silje Andreassen is funded by the Norwegian Multiregional Health Authorities through the BorrSci project (Lyme borreliosis; a scientific approach to reduce diagnostic and therapeutic uncertainties, project 2015113). Postdoctoral fellowship for Åslaug R. Lorentzen is funded by the South-Eastern Norway Regional Health Authority (project 2013089).

\section{Compliance with ethical standards}

Conflict of interest Mona $\mathrm{K}$ Beyer received minor grants or speaker's fees from Biogen Idec, Merck and Novartis. Hanne F. Harbo received minor grants or speaker's fees from Biogen, Sanofi Genzyme, Merck, Novartis and Teva. The other authors do not report any disclosures.

Open Access This article is distributed under the terms of the Creative Commons Attribution 4.0 International License (http:// creativecommons.org/licenses/by/4.0/), which permits unrestricted use, distribution, and reproduction in any medium, provided you give appropriate credit to the original author(s) and the source, provide a link to the Creative Commons license, and indicate if changes were made.

\section{References}

1. Hildenbrand P, Craven DE, Jones R, Nemeskal P (2009) Lyme neuroborreliosis: manifestations of a rapidly emerging zoonosis. AJNR Am J Neuroradiol 30(6):1079-1087

2. Stanek G, Wormser GP, Gray J, Strle F (2012) Lyme borreliosis. Lancet 379(9814):461-473

3. Rupprecht TA, Koedel U, Fingerle V, Pfister HW (2008) The pathogenesis of Lyme neuroborreliosis: from infection to inflammation. Mol Med 14(3-4):205-212

4. Halperin JJ (2015) Nervous system Lyme disease. Infect Dis Clin North Am 29(2):241-253

5. Pachner AR, Steiner I (2007) Lyme neuroborreliosis: infection, immunity, and inflammation. Lancet Neurol 6(6):544-552

6. Stanek G, Strle F (2008) Lyme disease: European perspective. Infect Dis Clin North Am 22(2):327-339 vii

7. Ogrinc K, Lusa L, Lotrič-Furlan S et al (2016) Course and outcome of early European Lyme neuroborreliosis (Bannwarth syndrome): clinical and laboratory findings. Clin Infect Dis 63(3):346-353

8. Schwenkenbecher P, Pul R, Wurster U et al (2017) Common and uncommon neurological manifestations of neuroborreliosis leading to hospitalization. BMC Infect Dis 17(1):90

9. Träisk F, Lindquist L (2012) Optic nerve involvement in Lyme disease. Curr Opin Ophthalmol 23(6):485-490

10. Mygland A, Ljøstad U, Fingerle V et al (2010) EFNS guidelines on the diagnosis and management of European Lyme neuroborreliosis. Eur J Neurol 17(1):8-16 e1-4

11. Eikeland R, Ljøstad U, Mygland A, Herlofson K, Lohaugen GC (2012) European neuroborreliosis: neuropsychological findings 30 months post-treatment. Eur J Neurol 19(3):480-487

12. Ljøstad U, Mygland A (2010) Remaining complaints 1 year after treatment for acute Lyme neuroborreliosis; frequency, pattern and risk factors. Eur J Neurol 17(1):118-123

13. Wormser GP, Dattwyler RJ, Shapiro ED et al (2006) The clinical assessment, treatment, and prevention of Lyme disease, human granulocytic anaplasmosis, and babesiosis: clinical practice guidelines by the infectious diseases society of America. Clin Infect Dis 43(9):1089-1134

14. Marques AR (2015) Laboratory diagnosis of Lyme disease: advances and challenges. Infect Dis Clin North Am 29(2):295-307

15. Cutler SJ, Ruzic-Sabljic E, Potkonjak A (2017) Emerging borreliae - expanding beyond Lyme borreliosis. Mol Cell Probes 31:22-27

16. Ramesh G, Didier PJ, England JD et al (2015) Inflammation in the pathogenesis of Lyme neuroborreliosis. Am J Pathol 185(5):1344 1360

17. Hansen K, Crone C, Kristoferitsch W (2013) Lyme neuroborreliosis. Handb Clin Neurol 115:559-575

18. Agarwal R, Sze G (2009) Neuro-Lyme disease: MR imaging findings. Radiology 253(1):167-173

19. Oksi J, Kalimo H, Marttila RJ et al (1996) Inflammatory brain changes in Lyme borreliosis. A report on three patients and review of literature. Brain 119(Pt 6):2143-2154 
20. Rafto SE, Milton WJ, Galetta SL, Grossman RI (1990) Biopsyconfirmed CNS Lyme disease: MR appearance at 1.5 T. AJNR Am J Neuroradiol 11(3):482-484

21. Halperin JJ, Luft BJ, Anand AK et al (1989) Lyme neuroborreliosis: central nervous system manifestations. Neurology 39(6):753-759

22. Halperin JJ, Pass HL, Anand AK, Luft BJ, Volkman DJ, Dattwyler RJ (1988) Nervous system abnormalities in Lyme disease. Ann N Y Acad Sci 539:24-34

23. Agosta F, Rocca MA, Benedetti B, Capra R, Cordioli C, Filippi M (2006) MR imaging assessment of brain and cervical cord damage in patients with neuroborreliosis. AJNR Am J Neuroradiol 27(4): 892-894

24. Fernandez RE, Rothberg M, Ferencz G, Wujack D (1990) Lyme disease of the CNS: MR imaging findings in 14 cases. AJNR Am J Neuroradiol 11(3):479-481

25. Krüger H, Heim E, Schuknecht B, Scholz S (1991) Acute and chronic neuroborreliosis with and without CNS involvement: a clinical, MRI, and HLA study of 27 cases. J Neurol 238(5):271280

26. Logigian EL, Johnson KA, Kijewski MF et al (1997) Reversible cerebral hypoperfusion in Lyme encephalopathy. Neurology 49(6): $1661-1670$

27. Morgen K, Martin R, Stone RD et al (2001) FLAIR and magnetization transfer imaging of patients with post-treatment Lyme disease syndrome. Neurology 57(11):1980-1985

28. Aalto A, Sjöwall J, Davidsson L, Forsberg P, Smedby O (2007) Brain magnetic resonance imaging does not contribute to the diagnosis of chronic neuroborreliosis. Acta Radiol 48(7):755-762

29. Akbik F, Matiello M, Piquet A, Cho T, Cohen A, Venna N (2017) Bibrachial plegia due to Lyme radiculopoliomyelitis-myelitis. J Neurol Sci 378:1-2

30. Haene A, Tröger M (2009) Diffuse hyperintense brainstem lesions in neuroborreliosis. Neurology 73(4):326

31. Kalina P, Decker A, Kornel E, Halperin JJ (2005) Lyme disease of the brainstem. Neuroradiology 47(12):903-907

32. Massengo SA, Bonnet F, Braun C, Vital A, Beylot J, Bastard J (2005) Severe neuroborreliosis: the benefit of prolonged highdose combination of antimicrobial agents with steroids-an illustrative case. Diagn Microbiol Infect Dis 51(2):127-130

33. Steinbach JP, Melms A, Skalej M, Dichgans J (2005) Delayed resolution of white matter changes following therapy of $\mathrm{B}$ burgdorferi encephalitis. Neurology 64(4):758-759

34. Tullman MJ, Delman BN, Lublin FD, Weinberger J (2003) Magnetic resonance imaging of meningoradiculomyelitis in early disseminated Lyme disease. J Neuroimaging 13(3):264-268

35. Chehrenama M, Zagardo MT, Koski CL (1997) Subarachnoid hemorrhage in a patient with Lyme disease. Neurology 48(2):520-523

36. Demaerel P, Crevits I, Casteels-Van Daele M, Baert AL (1998) Meningoradiculitis due to borreliosis presenting as low back pain only. Neuroradiology 40(2):126-127

37. Hattingen E, Weidauer S, Kieslich M, Boda V, Zanella FE (2004) MR imaging in neuroborreliosis of the cervical spinal cord. Eur Radiol 14(11):2072-2075

38. Lell M, Schmid A, Stemper B, Maihöfner C, Heckmann JG, Tomandl BF (2003) Simultaneous involvement of third and sixth cranial nerve in a patient with Lyme disease. Neuroradiology 45(2): 85-87

39. Mantienne C, Albucher JF, Catalaa I, Sévely A, Cognard C, Manelfe C (2001) MRI in Lyme disease of the spinal cord. Neuroradiology 43(6):485-488

40. Nelson JA, Wolf MD, Yuh WT, Peeples ME (1992) Cranial nerve involvement with Lyme borreliosis demonstrated by magnetic resonance imaging. Neurology 42(3 Pt 1):671-673

41. Savas R, Sommer A, Gueckel F, Georgi M (1997) Isolated oculomotor nerve paralysis in Lyme disease: MRI. Neuroradiology 39(2):139-141
42. Schneider A, Pischinger B, Wimmer S, Topakian R (2017) Anterocollis and startle myoclonus due to Lyme meningomyeloradiculitis. Acta Neurol Belg 117(1):317-318

43. Vanzieleghem B, Lemmerling M, Carton D et al (1998) Lyme disease in a child presenting with bilateral facial nerve palsy: MRI findings and review of the literature. Neuroradiology 40(11):739742

44. Back T, Grünig S, Winter Y et al (2013) Neuroborreliosisassociated cerebral vasculitis: long-term outcome and healthrelated quality of life. J Neurol 260(6):1569-1575

45. Defer G, Levy R, Brugiéres P, Postic D, Degos JD (1993) Lyme disease presenting as a stroke in the vertebrobasilar territory: MRI. Neuroradiology 35(7):529-531

46. Heinrich A, Khaw AV, Ahrens N, Kirsch M, Dressel A (2003) Cerebral vasculitis as the only manifestation of Borrelia burgdorferi infection in a 17-year-old patient with basal ganglia infarction. Eur Neurol 50(2):109-112

47. Klingebiel R, Benndorf G, Schmitt M, von Moers A, Lehmann R (2002) Large cerebral vessel occlusive disease in Lyme neuroborreliosis. Neuropediatrics 33(1):37-40

48. Lebas A, Toulgoat F, Saliou G, Husson B, Tardieu M (2012) Stroke due to Lyme neuroborreliosis: changes in vessel wall contrast enhancement. J Neuroimaging 22(2):210-212

49. Li S, Vytopil M, Hreib K, Craven DE (2015) Lyme disease presenting as multiple ischaemic strokes. Pract Neurol 15(4):284-288

50. May EF, Jabbari B (1990) Stroke in neuroborreliosis. Stroke 21(8): $1232-1235$

51. Romi F, Kråkenes J, Aarli JA, Tysnes OB (2004) Neuroborreliosis with vasculitis causing stroke-like manifestations. Eur Neurol 51(1):49-50

52. Schmiedel J, Gahn G, von Kummer R, Reichmann H (2004) Cerebral vasculitis with multiple infarcts caused by Lyme disease. Cerebrovasc Dis 17(1):79-81

53. Seelen M, van den Wijngaard IR, Rundervoort RS (2017) Cerebral vasculitis and stroke due to Lyme neuroborreliosis: a favorable clinical outcome after early antibiotic treatment. J Neurol Sci 375:165-166

54. Buchwald F, Abul-Kasim K, Tham J, Hansen BU (2010) Fatal course of cerebral vasculitis induced by neuroborreliosis. Neurol India 58(1):139-141

55. Jacobi C, Schwark C, Kress B, Hug A, Storch-Hagenlocher B, Schwaninger M (2006) Subarachnoid hemorrhage due to Borrelia burgdorferi-associated vasculitis. Eur J Neurol 13(5):536-538

56. Seijo Martinez M, Grandes Ibáñez J, Sánchez Herrero J, GarcíaMoncó JC (2001) Spontaneous brain hemorrhage associated with Lyme neuroborreliosis. Neurologia 16(1):43-45

57. Akgoz A, Mukundan S, Lee TC (2012) Imaging of rickettsial, spirochetal, and parasitic infections. Neuroimaging Clin N Am 22(4):633-657

58. Fallon BA, Lipkin RB, Corbera KM et al (2009) Regional cerebral blood flow and metabolic rate in persistent Lyme encephalopathy. Arch Gen Psychiatry 66(5):554-563

59. Newberg A, Hassan A, Alavi A (2002) Cerebral metabolic changes associated with Lyme disease. Nucl Med Commun 23(8):773-777

60. Donta ST, Noto RB, Vento JA (2012) SPECT brain imaging in chronic Lyme disease. Clin Nucl Med 37(9):e219-e222

61. Fallon BA, Das S, Plutchok JJ, Tager F, Liegner K, Van Heertum R (1997) Functional brain imaging and neuropsychological testing in Lyme disease. Clin Infect Dis 25(Suppl 1):S57-S63

62. Fallon BA, Keilp J, Prohovnik I, Heertum RV, Mann JJ (2003) Regional cerebral blood flow and cognitive deficits in chronic Lyme disease. J Neuropsychiatr Clin Neurosci 15(3):326-332

63. Lummel N, Koch M, Klein M, Pfister HW, Brückmann H, Linn J (2016) Spectrum and prevalence of pathological intracranial magnetic resonance imaging findings in acute bacterial meningitis. Clin Neuroradiol 26(2):159-167 
64. Splendiani A, Puglielli E, De Amicis R, Necozione S, Masciocchi C, Gallucci M (2005) Contrast-enhanced FLAIR in the early diagnosis of infectious meningitis. Neuroradiology 47(8):591-598

65. Parmar H, Sitoh YY, Anand P, Chua V, Hui F (2006) Contrastenhanced flair imaging in the evaluation of infectious leptomeningeal diseases. Eur J Radiol 58(1):89-95

66. Strle F, Stanek G (2009) Clinical manifestations and diagnosis of Lyme borreliosis. Curr Probl Dermatol 37:51-110

67. Gupta S, Mends F, Hagiwara M, Fatterpekar G, Roehm PC (2013) Imaging the facial nerve: a contemporary review. Radiol Res Pract 2013:248039

68. Lim HK, Lee JH, Hyun D et al (2012) MR diagnosis of facial neuritis: diagnostic performance of contrast-enhanced 3D-FLAIR technique compared with contrast-enhanced 3D-T1-fast-field echo with fat suppression. AJNR Am J Neuroradiol 33(4):779-783

69. Berlit P, Pohlmann-Eden B, Henningsen H (1991) Brown-sequard syndrome caused by Borrelia burgdorferi. Eur Neurol 31(1):18-20

70. Charles V, Duprez TP, Kabamba B, Ivanoiu A, Sindic CJ (2009) Poliomyelitis-like syndrome with matching magnetic resonance features in a case of Lyme neuroborreliosis. BMJ Case Rep 2009. https://doi.org/10.1136/bcr.07.2008.0527

71. De Cauwer H, Declerck S, De Smet J et al (2009) Motor neuron disease features in a patient with neuroborreliosis and a cervical anterior horn lesion. Acta Clin Belg 64(3):225-227

72. Dryden MS, O'Connell S, Samuel W, Iannotti F (1996) Lyme myelitis mimicking neurological malignancy. Lancet 348(9027):624

73. Kohler J (1989) Lyme borreliosis: a case of transverse myelitis with syrinx cavity. Neurology 39(11):1553-1554

74. Lesca G, Deschamps R, Lubetzki C, Levy R, Assous M (2002) Acute myelitis in early Borrelia burgdorferi infection. J Neurol 249(10):1472-1474

75. Meurs L, Labeye D, Declercq I, Piéret F, Gille M (2004) Acute transverse myelitis as a main manifestation of early stage II neuroborreliosis in two patients. Eur Neurol 52(3):186-188

76. Walid MS, Ajjan M, Ulm AJ (2008) Subacute transverse myelitis with Lyme profile dissociation. Ger Med Sci 6:Doc04
77. Martin R, Meinck HM, Schulte-Mattler W, Ricker K, Mertens HG (1990) Borrelia burgdorferi myelitis presenting as a partial stiff man syndrome. J Neurol 237(1):51-54

78. Grill MF (2018) Infectious myelopathies. Continuum (Minneap Minn) 24(2, Spinal Cord Disorders):441-473

79. Halperin JJ, Logigian EL, Finkel MF, Pearl RA (1996) Practice parameters for the diagnosis of patients with nervous system Lyme borreliosis (Lyme disease). Neurology 46(3):619-627

80. Gorson KC, Kolb DA, Marks DS, Hayes MT, Baquis GD (2011) Acute brachial diplegia due to Lyme disease. Neurologist 17(1):24-27

81. Chhabra A (2014) Peripheral MR neurography: approach to interpretation. Neuroimaging Clin N Am 24(1):79-89

82. Pittock SJ, Debruyne J, Krecke KN et al (2010) Chronic lymphocytic inflammation with pontine perivascular enhancement responsive to steroids (CLIPPERS). Brain 133(9):2626-2634

83. Bertrand A, Leclercq D, Martinez-Almoyna L, Girard N, Stahl JP, De-Broucker T (2017) MR imaging of adult acute infectious encephalitis. Med Mal Infect 47(3):195-205

84. Horger M, Beck R, Fenchel M et al (2012) Imaging findings in tickborne encephalitis with differential diagnostic considerations. AJR Am J Roentgenol 199(2):420-427

85. Lenhard T, Ott D, Jakob NJ et al (2016) Predictors, neuroimaging characteristics and long-term outcome of severe European tickborne encephalitis: a prospective cohort study. PLoS One 11(4): e0154143

86. Garkowski A, Zajkowska J, Zajkowska A et al (2017) Cerebrovascular manifestations of Lyme neuroborreliosis-a systematic review of published cases. Front Neurol 8:146

\section{Publisher's Note}

Springer Nature remains neutral with regard to jurisdictional claims in published maps and institutional affiliations. 